\title{
Different dynamical histories for comets C/2001 Q4 and C/2002 T7?
}

\author{
M. Królikowska ${ }^{1}$, P. A. Dybczyński ${ }^{2}$, and G. Sitarski ${ }^{1}$
}

\author{
1 Space Research Centre of the Polish Academy of Sciences, Bartycka 18A, 00-716 Warsaw, Poland \\ e-mail: [mkr; sitarski]@cbk.waw.pl \\ 2 Astronomical Observatory Institute, Faculty of Physics, A. Mickiewicz University, Słoneczna 36, 60-286 Poznań, Poland \\ e-mail: dybol@amu.edu.pl
}

Received 13 April 2012 / Accepted 23 June 2012

\begin{abstract}
Context. Both, C/2001 Q4 and C/2002 T7, are widely regarded in the literature as dynamically new comets that are visiting our planetary system for the first time from the Oort Cloud.

Aims. We study the past dynamical evolution of these two bright comets that both have an original semimajor axis inside the so-called Oort spike (i.e. with $1 / a_{\text {ori }}<10^{-4} \mathrm{AU}^{-1}$ ).

Methods. For each comet, we constructed a dedicated grid of independent, starting, osculating swarms of 5000 orbits based on different subsets of positional data and different dependences of the non-gravitational acceleration on the heliocentric distance. We then followed numerically each swarm of non-gravitational orbits one orbital revolution into the past, taking into account both planetary and Galactic perturbations and checking for all known stellar perturbers. This method allows us to obtain the orbital elements and their uncertainties at the previous perihelion passage.

Results. We find that the dedicated $g(r)$-like function seems to be more adequate for describing the non-gravitational effects than the standard $g(r)$-function in the motion of both comets, but we are able to estimate only two parameters: the scale distance $r_{0}$, and the exponent $m$. We show, however, that the greatest change in the previous perihelion value relative to that obtained in the standard approach results from the type of data subset used for non-gravitational orbit determination. The form of the dependence of nongravitational acceleration on heliocentric distance is of secondary importance for both investigated comets in this context. We find that only comet C/2002 T7 passed far beyond the planetary system during its previous perihelion passage and that C/2001 Q4 was probably well inside the Saturn orbit at a previous perihelion.

Conclusions. We argue that for these comets (which have long sequences of positional data), the safest method for the previous perihelion determination is to exclude data within time intervals where some local outbursts were reported. We recommend that the non-gravitational models based on data taken at larger perihelion distance are more appropriate for estimating the distance of previous perihelion passage of C/2001 Q4 and those based on a pre-perihelion data set for previous perihelion estimation of C/2002 T7. These models suggest that $\mathrm{C} / 2001 \mathrm{Q} 4$ passed its previous perihelion closer than 6-7 AU from the Sun, so is dynamically old, whereas $\mathrm{C} / 2002 \mathrm{~T} 7$ at a distance larger than $400 \mathrm{AU}$, is a dynamically new comet since it overcame the Jupiter-Saturn barrier.
\end{abstract}

Key words. comets: general - comets: individual: C/2001 Q4 - comets: individual: C/2002 T7 - Oort Cloud

\section{Introduction}

Comets C/2001 Q4 NEAT and C/2002 T7 LINEAR were discovered at the large heliocentric distances of $r=10.1 \mathrm{AU}$ (in August 2001) and $r=6.9 \mathrm{AU}$ (in October 2002), respectively. At the moment of its discovery, C/2001 Q4 was a 20 mag object that showed a $8^{\prime \prime}$ coma, whereas the coma of C/2002 T7 (17.5 mag at the moment of discovery) was only marginally greater than the Airy disks of nearby stars (Kammerer 2011). In addition, Tozzi et al. (2003) observed the coma of comet C/2001 Q4 at a large distance before perihelion (8.6 AU from the Sun). They reported a conic structure in the northern hemisphere of its coma and interpreted this structure as a dust fan produced by an active region located at the nucleus. Both objects passed relatively close to the Earth in May 2003, C/2001 Q4 at the distance of $0.321 \mathrm{AU}$ (48 million kilometers) and C/2002 T7 at the distance of $0.266 \mathrm{AU}$ (40 million kilometers). They reached naked-eye visibility a year later, in April-May 2004, when they were close to their perihelia. Comet C/2002 T7 passed perihelion at the distance of about $0.61 \mathrm{AU}$ from the Sun reaching its maximum apparent brightness of mag 2.7 about a month later. Comet C/2001 Q4 was fainter, but still brighter than 4 mag, during its perihelion passage at a distance of about 0.96 AU from the Sun. For observers in the northern hemisphere at mid-latitudes, there was the unusual phenomenon of seeing two bright comets in the same direction in the evening sky: since the middle of the twentieth century, this was indeed the only documented case of two comets brighter than fourth magnitude being observed at the same time. Observational statistics tell us that we should be able to view a comet of fourth magnitude or brighter once every two years ${ }^{1}$.

It is therefore unsurprising that these two comets were intensively observed not only visually but also in other spectral regimes, mostly during observational campaigns dedicated to both comets, e.g. Hogerheijde et al. (2009); Combi et al. (2009); Buffington et al. (2008); Gibb et al. (2007); Milam et al. (2006). Both C/2001 Q4 NEAT and C/2002 T7 LINEAR are nearly parabolic comets, hence were also compared with each other in the context of their dynamical histories, both being widely regarded as dynamically new comets. They are also included in the sample of long-period (LP) comets in Earth-crossing or

\footnotetext{
1 See for example:

http://www.icq.eps.harvard.edu/brightest.html
} 
Table 1. Observational material for the two comets studied in this paper.

\begin{tabular}{lcccc}
\hline \hline Name & $\begin{array}{c}\text { Paper I } \\
\text { interval of obs. }\end{array}$ & No. of obs. & Present investigation \\
& interval of obs. & No. of obs. \\
\hline C/2001 Q4 NEAT & 24 August 2001-18 August 2006 & 2661 & 24 August 2001-18 August 2006 & 2681 \\
C/2002 T7 LINEAR & 12 October 2002-7 March 2006 & 4451 & 12 October 2002-20 March 2006 & 4507 \\
\hline
\end{tabular}

Notes. Astrometric data were taken on 14 December 2011 from the IAU Minor Planet Center Web Page http://www. minorplanetcenter.net/db_search.
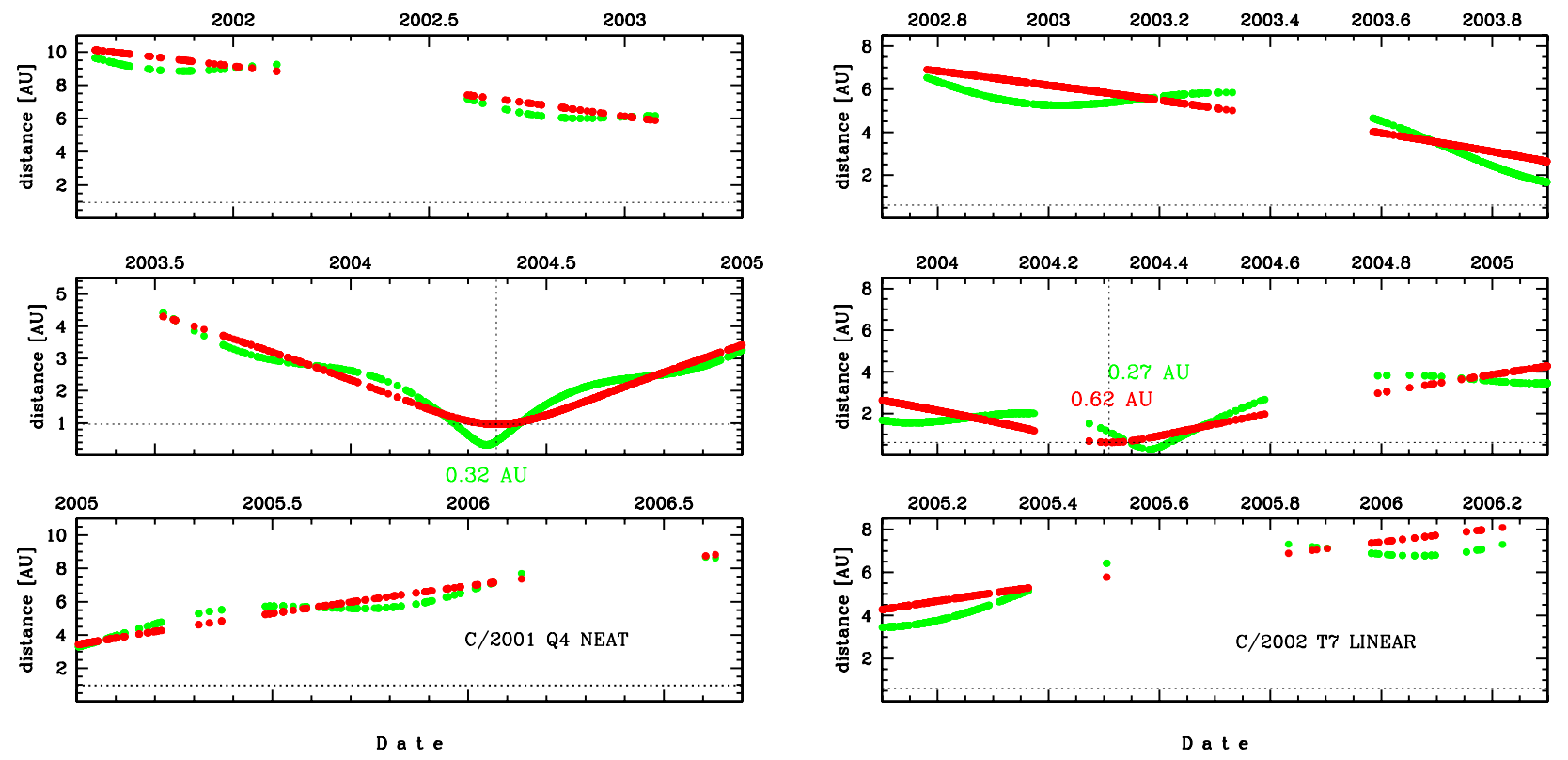

Fig. 1. The distance from the Sun (red/black dots) and from the Earth (green/grey dots) for comets C/2001 Q4 NEAT (left panel) and C/2002 T7 LINEAR (right panel) for all available observations.

approaching orbits analysed by Fernández \& Sosa (2012). These authors, however, use only pure gravitational orbits and simplify distinction between dynamically old and new comets. Therefore, from these authors' point of view, these two comets are rather indistinguishable. It seems, however, that one of them is a dynamically new comet (C/2002 T7) while the other (C/2001 Q4) visited the inner part of the solar system during its previous perihelion passage (Królikowska \& Dybczyński 2010, hereafter Paper I). In Paper I, these two comets were included into a group of five comets for which some systematic deviations in the $\mathrm{O}-\mathrm{C}$ (observed minus calculated value) time variations were detected in the case of the non-gravitational (hereafter NG) motion, despite the significant improvements that have been achieved in the orbit determinations based on the standard type of NG effects. These improvements were measured by three factors: (i) root mean square (rms) decreasing, (ii) weaker systematic trends in the $\mathrm{O}-\mathrm{C}$ time variations, and (iii) the closer similarity of the $\mathrm{O}-\mathrm{C}$ distribution to a normal one. We then concluded that these comets require additional investigation, perhaps based on a model with a different $g(r)$-dependence on the heliocentric distance, $r$, than the standard one, which was originally proposed by Marsden et al. (1973) and is commonly used in cometary dynamics. In the present investigation, we show that regardless of the assumed $g(r)$-function, a general conclusion about the different dynamic statuses of these two comets seems to be valid.

Both comets were observed astrometrically for a long time: comet C/2001 Q4 for five years from 24 August 2001 to 18 August 2006 (Table 1), i.e. from a heliocentric distance of 10.1 AU before perihelion to $8.8 \mathrm{AU}$ after perihelion, and the comet $\mathrm{C} / 2002 \mathrm{~T} 7$ for almost 3.5 years over the period from 12 October 2002 (6.9 AU) to 20 March 2006 (8.1 AU). Figure 1 shows how the astrometric data of both comets are distributed within the observational periods, in connection with their heliocentric and geocentric distances.

It is well-known that both objects show clear deviations from purely gravitational motion (Marsden \& Williams 2008, and Table 2). This means that, assuming standard NG model motion, the noticeably closer orbital fitting to astrometric observations could be reached (measured by a decrease in rms, the $\mathrm{O}-\mathrm{C}$ time variations, and the degree of similarity of the $\mathrm{O}-\mathrm{C}$ distribution to a normal distribution; see also Paper I) than by a pure gravitational (hereafter GR) model. Thus, the two comets are especially well-suited to testing the methods of the original NG orbit determinations. Unfortunately, the methods proposed here can be used today only for a small sample of known one-apparition comets, because these studies need a clear presence of NG effects in the cometary motion and a long sequence of astrometric observations covering a wide range of heliocentric distances. Nowadays, more and more LP comets are discovered long before their perihelion passages and are followed on the outgoing legs of their orbits to large heliocentric distances, so each year the number of good candidates for this purpose increases.

The main goal of this paper is to investigate the dynamical pasts of C/2001 Q4 and C/2002 T7 by analysing different sequences of their astrometric data, as well as different NG models. In particular, we present two types of sub-sequences of data that seem more suitable for study of the original NG orbits of two investigated comets (Sect. 3.1) than all the available astrometric 
Table 2. Original and future semimajor axes derived from pure gravitational (GR) nominal solutions (Cols. 2, 3) and non-gravitational (NG) nominal solutions (Cols. 4,5$)$, where all observations were taken into account and standard $g(r)$ function for NG effects were used.

\begin{tabular}{|c|c|c|c|c|c|c|c|c|c|}
\hline \multirow{3}{*}{$\begin{array}{l}\text { Name } \\
1\end{array}$} & \multicolumn{2}{|c|}{ GR solutions } & \multicolumn{2}{|c|}{ NG solutions } & \multirow{3}{*}{$\begin{array}{c}\mathrm{rms}_{\mathrm{GR}} \\
\quad " \\
6\end{array}$} & \multirow{3}{*}{$\begin{array}{c}\mathrm{rms}_{\mathrm{NG}} \\
\quad ! \\
7\end{array}$} & \multicolumn{2}{|c|}{ Number of } & \multirow[t]{2}{*}{ Ref. } \\
\hline & $\begin{array}{l}1 / a_{\text {ori }} \\
\text { in units of }\end{array}$ & $\begin{array}{c}1 / a_{\mathrm{fut}} \\
10^{-6} \mathrm{AU}^{-1}\end{array}$ & $\begin{array}{l}1 / a_{\text {ori }} \\
\text { in units of }\end{array}$ & $\begin{array}{c}1 / a_{\mathrm{fut}} \\
10^{-6} \mathrm{AU}^{-1}\end{array}$ & & & & $\begin{array}{l}\text { res. } \\
\text { NG }\end{array}$ & \\
\hline & 2 & 3 & 4 & 5 & & & 8 & 9 & 10 \\
\hline \multirow[t]{3}{*}{ C/2001 Q4 NEAT } & $+14.82 \pm 0.41$ & $-729.21 \pm 0.41$ & $+60.56 \pm 0.81$ & $-694.40 \pm 0.46$ & 1.25 & 0.56 & 5347 & 5316 & present \\
\hline & $+12.51 \pm 0.43$ & $-731.74 \pm 0.43$ & $+60.87 \pm 0.48$ & $-696.62 \pm 0.48$ & 1.29 & 0.63 & 5305 & 5263 & PIw \\
\hline & $+05.80 \pm 0.44$ & $-738.46 \pm 0.44$ & $+59.59 \pm 0.70$ & $-696.74 \pm 0.64$ & 1.69 & 0.80 & 5223 & 5223 & PI \\
\hline \multirow[t]{3}{*}{ C/2002 T7 LINEAR } & $-11.17 \pm 0.18$ & $-650.89 \pm 0.18$ & $+25.65 \pm 0.34$ & $-713.74 \pm 1.20$ & 0.55 & 0.50 & 8796 & 8878 & present \\
\hline & $-13.84 \pm 0.16$ & $-653.57 \pm 0.16$ & $+20.72 \pm 0.39$ & $-660.08 \pm 1.05$ & 0.61 & 0.58 & 8596 & 8768 & PIw \\
\hline & $-24.42 \pm 0.24$ & $-664.14 \pm 0.24$ & $+19.73 \pm 0.39$ & $-650.86 \pm 1.03$ & 1.21 & 0.64 & 8643 & 8643 & PI \\
\hline
\end{tabular}

Notes. The rms's and number of residuals are given in Cols. 6, 7 and 8, 9, respectively. There are three rows for each comet: the first describes present results with the weighting of the data, and the second and third rows reproduce the results given in Paper I, where PIw and PI (last column) represent models with and without weighting, respectively.

material. These are models based on data from the incoming branches of orbits, and models based on data from two orbital legs but taken only at large distances from the Sun, when the activity of a comet is relatively less important than around perihelion and can be described in a predictable manner.

In Sect. 2, we briefly describe our standard method of modelling the evolution of actual LPCs and the previous results obtained for C/2001 Q4 and C/2002 T7. In Sect. 3, we propose a new approach to investigating the past evolution of these two comets, which have long sequences of astrometric data and manifest easily detectable NG effects. In Sect. 4, we present new results based on this new approach. We then explore a grid of models using different criteria for the astrometric data compilation, as well as different models of the NG acceleration. In Sect. 5, we discuss which models are the most appropriate for investigating the previous perihelion passages for these particular comets and draw general conclusions from our investigation.

\section{Previous approach to determining the dynamical statuses of C/2001 Q4 NEAT and C/2002 T7 LINEAR}

Long intervals of data make it possible to determine the osculating orbits of both comets with a very high precision (very small uncertainties) within the a priori adopted standard type of NG model of motion (Marsden et al. 1973). Such results were described in Paper I, where we used a dedicated Monte Carlo method to control the propagation of uncertainties in the past and future motions of these comets. We thus generated the swarm of osculating elements of virtual comets (VCs) that are wellfitted to the observations (Sitarski 1998) and then propagated them to both the previous and subsequent (i.e. next) perihelion passages, taking into account both planetary and Galactic perturbations. Our method of numerical calculations is described in detail in Paper I. For both comets, we derived very accurate results, where swarms of VC orbital elements are welldescribed by Gaussian distributions both for previous orbits at previous perihelion passages and future hyperbolic orbits derived at $120000 \mathrm{AU}$ from the Sun. Although past swarms of both comets are rather condensed they differ dramatically in terms of their previous perihelion distances. Comet C/2001 Q4 was at its previous perihelion close to the Sun $(1.9 \mathrm{AU} \pm 0.1 \mathrm{AU})$ about 2 million years ago, whereas C/ 2002 T7 passed its previous perihelion at a distance of $\sim 140 \mathrm{AU} \pm 20 \mathrm{AU}$ from the Sun more than 10 million years ago. We concluded that only C/2002 T7 seems to be dynamically new. We also discussed the problem of other forms of NG accelerations than that based on standard $g(r)$ for these two comets. We estimated that differences in osculating orbital elements among the considered NG models for these two comets were about one order of magnitude smaller than those obtained between GR and NG models. We therefore found that the main conclusion about dynamical statuses of these comets remains unchanged.

These results were obtained with the assumption that the NG osculating solution based on all of the available observational material is always a better starting orbit when deriving the actual past and future motions of long-period comets than the NG starting orbit derived from some subset of existing astrometric data. For the majority of long-period comets, this is true because usually we have a limited sequence of astrometric data, as well as modest information about their photometric behaviour. Thus, a significantly smaller subset of data did not allow us to determine the NG effects with sufficient credibility. However, for a few LP comets with small perihelion distances we have very long runs of observations that allow us to exclude data within time intervals where outbursts or any other strange cometary behaviour were reported (that usually occurred around perihelion passage). We have such a situation in the case of two comets considered in the present paper. In what follows, we show that some subsets of data seem to be more adequate than a full data set for determining the starting osculating orbit for our purposes, i.e. for the original orbit determination and previous perihelion-passage dynamical calculations.

Additionally, we found that the observational material had been corrected and supplemented in the past few years (after our previous investigations). We noted that some observations had been removed from the publicly available database and some new data were added. All results presented in this paper are based on data retrieved from the IAU Minor Planet Center on December 14, 2011. The differences in the global characteristics between the previous and current data sets are given in Table 1.

\section{New approach}

To identify the best method for determining an original orbit to study the past dynamical evolution of these two comets, we explore grids of models with different ways of constructing data samples (Sect. 3.1) and different forms of NG acceleration (Sect. 3.2). To this aim, we tried to obtain the most appropriate initial NG osculating orbit (nominal orbit). As in our previous investigations (e.g. Paper I), we then constructed a swarm 
Table 3. Grid of models for C/2001 Q4 NEAT.

\begin{tabular}{|c|c|c|c|c|c|}
\hline $\begin{array}{l}\text { Row } \\
\text { No. }\end{array}$ & & Models based on all data & $\begin{array}{l}\text { Models based on all data } \\
\text { at large distances } \\
(r>3.0 \mathrm{AU})\end{array}$ & $\begin{array}{l}\text { Models based on all data } \\
\text { at large distances } \\
\text { agreed with Combi et al. (2009) }\end{array}$ & $\begin{array}{l}\text { Models based on all data } \\
\text { before perihelion }\end{array}$ \\
\hline [1] & type of data subsets & ALL & DIST1 & DIST2 & PRE \\
\hline [2] & interval of observations & 24 Aug. $2001-18$ Aug. 2006 & 24 Aug. 2001-18 Aug. 2006 & 24 Aug. 2001-18 Aug. 2006 & 24 Aug. $2001-15$ April 2004 \\
\hline [3] & interval of ejected data & - & 9 Nov. 2003-23 Nov. 2004 & 15 April 2004-25 July 2004 & after 15 April 2004 \\
\hline [4] & Number of observations & 2681 & 692 & 1518 & 525 \\
\hline [5] & Number of residuals & 5316 & 1369 & 3012 & 1030 \\
\hline \multicolumn{6}{|c|}{ NG model type: STD } \\
\hline [6] & rms [arcsec] & 0.56 & 0.59 & 0.52 & 0.38 \\
\hline [7] & $\chi^{2} /$ probability & $31.17 / 0.0028$ & $21.45 / 0.257$ & $19.02 / 0.391$ & $16.00 / 0.593$ \\
\hline [8] & $1 / a_{\text {ori }}\left[10^{-6} \mathrm{AU}^{-1}\right]$ & $60.6 \pm 0.8$ & $36.2 \pm 1.7$ & $49.3 \pm 1.0$ & $33.3 \pm 0.9$ \\
\hline [9] & $1 / a_{\text {fut }}\left[10^{-6} \mathrm{AU}^{-1}\right]$ & $-694.4 \pm 0.5$ & $-676.4 \pm 2.9$ & & \\
\hline [10] & $q_{\mathrm{prev}}[\mathrm{AU}]$ & $1.89 \pm 0.05$ & $13.0_{-2.7}^{+3.3}$ & $3.35_{-0.22}^{+0.25}$ & $20.4_{-2.8}^{+3.3}$ \\
\hline \multicolumn{6}{|c|}{ NG model type: GEN } \\
\hline$\left[\begin{array}{l}{[11]} \\
{[12]}\end{array}\right.$ & $\begin{array}{l}\text { NG parameters } \\
\text { rms [arcsec] }\end{array}$ & $\begin{array}{c}r_{0}=4.0 \mathrm{AU} \\
0.56\end{array}$ & $\begin{array}{c}r_{0}=4.0 \mathrm{AU} ; m=-1.9 \\
0.57\end{array}$ & $\begin{array}{c}r_{0}=4.0 \mathrm{AU} ; m=-1.9 \\
0.51\end{array}$ & $\begin{array}{c}r_{0}=4.0 \mathrm{AU} ; m=-1.9 \\
0.38\end{array}$ \\
\hline$[13]$ & $1 / a_{\text {ori }}\left[10^{-6} \mathrm{AU}^{-1}\right]$ & $58.0 \pm 0.8$ & $36.9 \pm 1.7$ & $46.2 \pm 1.0$ & $28.9 \pm 0.9$ \\
\hline [14] & $q_{\mathrm{prev}}[\mathrm{AU}]$ & $2.08 \pm 0.07$ & $11.8_{-2.4}^{+3.3}$ & $4.24_{-0.33}^{+0.38}$ & $46.0_{-7.5}^{+9.4}$ \\
\hline \multicolumn{6}{|c|}{ NG model type: YAB } \\
\hline [15] & rms [arcsec] & 0.56 & 0.57 & 0.51 & greater than STD \\
\hline [16] & $1 / a_{\text {ori }}\left[10^{-6} \mathrm{AU}^{-1}\right]$ & $54.6 \pm 0.8$ & $36.27 \pm 1.7$ & $43.1 \pm 1.0$ & \\
\hline [17] & $q_{\text {prev }}[\mathrm{AU}]$ & $2.44 \pm 0.10$ & $12.8^{+3.7}$ & $5.63^{+0.61}$ & \\
\hline
\end{tabular}

Notes. Row [7] describes the result of $\chi^{2}$ test for sampling O-C distribution derived in a given type of data subset with 18 degrees of freedom in each case; first value represents the $\chi^{2}$-value, second $-\chi^{2}$ significance; for 18 degrees of freedom, the critical value of $\chi^{2}$ at a confidence level of 0.95 is equal to 28.87 .

of $5000 \mathrm{VCs}$ and then propagated each VC backward up to the distance of $250 \mathrm{AU}$ to obtain an original, nominal barycentric orbit (free from any planetary perturbations), together with its orbital element uncertainties.

\subsection{Data subsets}

For the starting osculating orbit determination, we use the following subsets of data:

- Data type: ALL - all observations. Results are based on all observations selected and weighted for standard NG model of motion.

- Data type: DIST - a subset of observations taken only at larger distances from the Sun. For comet C/2001 Q4, two types of distant-data subsets are constructed: DIST1 - when the observations around perihelion taken at a distance below 3.0 AU were omitted and DIST2 where a dedicated criterion based on the observed cometary unusual behaviour was used.

- Data type: PRE - a subset of pre-perihelion data only.

The respective observation intervals and number of observations in each data sample are given in Tables 3, 4 and Fig. 1. For each data subset, we first performed a rigorous data selection and weighting procedure using a standard NG force model. We then determined different variants of the osculating starting orbit by using different NG force models (see Sect. 3.2) and the same, already preselected and weighted data. This means that each set of astrometric data was processed individually for the standard model of NG acceleration (STD model, see Sect. 3.2), according to the method described in great detail in Paper I. The number of residuals used and rms derived for an osculating orbit determination in the standard NG model are given in the third column of
Tables 3, 4. The O-C diagrams obtained for all available astrometric observations (ALL data type) and the standard NG force model (STD) are shown in Fig. 2. One can see some evident trends in $\mathrm{O}-\mathrm{C}$ diagrams for models based on all observations (ALL type of data sets). Deviations of $\mathrm{O}-\mathrm{C}$ distributions from the Gaussian one were first analysed using a $\chi^{2}$ test; the probabilities that the obtained $\chi^{2}$-value represent the normal distribution are given in row [7] of Tables 3, 4 (for 18 degrees of freedom, the critical value of $\chi^{2}$ at a confidence level of 0.95 is equal to 28.87). One can see that the probability that $\mathrm{O}-\mathrm{C}$ distribution in an ALL type of model is normal is 0.028 and 0.0033 , for C/2001 Q4 and C/2002 T7, respectively (Col. 3 of Tables 3, 4). The deviations from a Gaussian distribution were also measured by the kurtosis and skewness, defined as $K=\frac{\mu_{4}}{\sigma^{4}}-3$ and $\gamma=\frac{\mu_{3}}{\sigma^{3}}$, respectively, where $\mu_{3}, \mu_{4}$ are the third and fourth central moments and $\sigma$ is the standard deviation. Qualitatively speaking, values of kurtosis and skewness close to zero indicate that the considered distribution is satisfactorily similar to a normal distribution. The kurtosis and skewness of $\mathrm{O}-\mathrm{C}$ distributions based on an ALL type of data differ substantially from zero, which additionally shows that these distributions are not Gaussian in these cases. This problem was pointed out in Paper I and was the main reason for examining other forms of $\mathrm{NG}$ accelerations or/and types of data samples to calculate the initial osculating orbit for a previous perihelion determination. However, applying other forms of NG forces (see Sect. 3.2) does not solve this problem satisfactory. The reason lies in the unusual activity at small perihelion distances (Sect. 4.2) that is impossible to model with a single set of NG parameters determined from all the astrometric data or at least consistent with these data. The situation is much better for the remaining types of data sets. A $\chi^{2}$ test shows that the $\mathrm{O}-\mathrm{C}$ distributions are Gaussian or almost Gaussian for NG models determined based on DIST and PRE subsets of data 
Table 4. Grid of models for C/2002 T7 LINEAR; row [7] as in Table 3.

\begin{tabular}{|c|c|c|c|c|}
\hline $\begin{array}{l}\text { Row } \\
\text { No. }\end{array}$ & & Models based on all data & $\begin{array}{l}\text { Models based on observations } \\
\text { at large distances }(r>3.0 \mathrm{AU})\end{array}$ & $\begin{array}{l}\text { Models based on all data } \\
\text { before perihelion }\end{array}$ \\
\hline [1] & type of data subsets & ALL & DIST & PRE \\
\hline [2] & interval of observations & 12 October 2002-20 March 2006 & 12 October 2002-20 March 2006 & 12 October 2002-17 April 2004 \\
\hline [3] & interval of ejected data & - & 26 October 2003-22 October 2004 & after 17 April 2004 \\
\hline [4] & Number of observations & 4507 & 2611 & 3655 \\
\hline [5] & Number of residuals & 8878 & 5141 & 7170 \\
\hline \multicolumn{5}{|c|}{ NG model type: STD } \\
\hline [6] & rms [arcsec] & 0.50 & 0.39 & 0.36 \\
\hline [7] & $\chi^{2} /$ probability & $38.49 / 0.0033$ & $25.81 / 0.104$ & $19.48 / 0.363$ \\
\hline [8] & $1 / a_{\text {ori }}\left[10^{-6} \mathrm{AU}^{-1}\right]$ & $25.6 \pm 0.4$ & $21.7 \pm 0.9$ & $17.7 \pm 0.5$ \\
\hline [9] & $1 / a_{\text {fut }}\left[10^{-6} \mathrm{AU}^{-1}\right]$ & $-713.7 \pm 1.2$ & $-676.4 \pm 2.9$ & \\
\hline [10] & $q_{\text {prev }}[\mathrm{AU}]$ & $30.0_{-3.4}^{+3.8}$ & $103_{-26}^{+35}$ & $405_{-76}^{+94}$ \\
\hline \multicolumn{5}{|c|}{ NG model type: GEN } \\
\hline [11] & NG parameters & $r_{0}=1.5 \mathrm{AU}$ & $r_{0}=1.5 \mathrm{AU}$ & $r_{0}=4.0 \mathrm{AU}$ \\
\hline [12] & rms $[\operatorname{arcsec}]$ & 0.47 & 0.38 & 0.35 \\
\hline$[13]$ & $1 / a_{\text {ori }}\left[10^{-6} \mathrm{AU}^{-1}\right]$ & $26.5 \pm 0.4$ & $22.6 \pm 0.9$ & $15.6 \pm 0.5$ \\
\hline [14] & $q_{\text {prev }}[\mathrm{AU}]$ & $23.1_{-2.5}^{+2.9}$ & $76_{-19}^{+26}$ & almost all (99.6\%) escaping \\
\hline \multicolumn{5}{|c|}{ NG model type: YAB } \\
\hline [15] & rms $[\operatorname{arcsec}]$ & greater than STD & 0.39 & 0.35 \\
\hline [16] & $1 / a_{\text {ori }}\left[10^{-6} \mathrm{AU}^{-1}\right]$ & - & $20.1 \pm 0.9$ & $14.9 \pm 0.5$ \\
\hline [17] & $q_{\mathrm{prev}}[\mathrm{AU}]$ & & $176_{-46}^{+65}$ & all escaping \\
\hline
\end{tabular}

Notes. Row [7] as in Table 3.
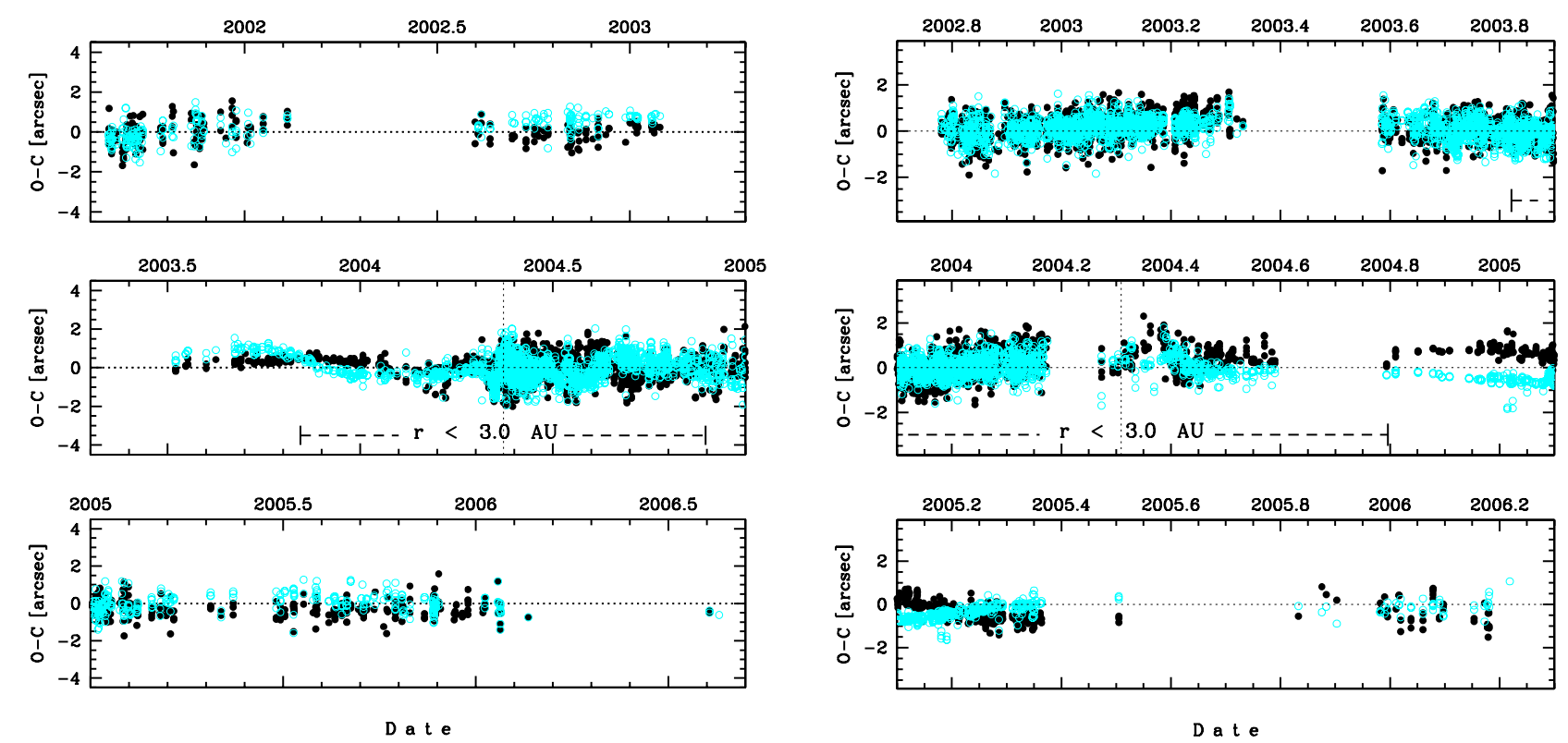

Fig. 2. The O-C diagrams for comets C/2001 Q4 NEAT (left panel) and C/2002 T7 LINEAR (right panel) for NG model of standard acceleration and all available data (model ALL+STD, see Sect. 3). The residuals in right ascension are shown as black dots and in declination as cyan/grey circles; the moment of perihelion passage is shown by a dashed vertical line, and a dashed horizontal line corresponds to regions where data were taken at small heliocentric distances $(r<3.0 \mathrm{AU})$.

where trends in the $\mathrm{O}-\mathrm{C}$ diagrams are also negligible as one can see in the last two columns of Tables 3, 4 and Fig. 4. For example, it seems possible to model motion of comet C/2002 T7 with two independent sets of three standard NG parameters. In Fig. 4, the observations are divided into two perihelion zones: data taken when the comet was further than 3.0 AU from the Sun (DIST type of data, indicated by both black dots and dark grey circles) and the rest of data around perihelion passage taken between 27 October 2003 and 16 October 2004 (in light grey). The last model, based on data taken closer than 3.0 AU from the Sun, is presented here only to show a complete picture of the $\mathrm{O}-\mathrm{C}$ diagram and is not used in any other analysis described in this paper for the obvious reason that we are looking for a previous perihelion passage. In our opinion, models based on a DIST type 


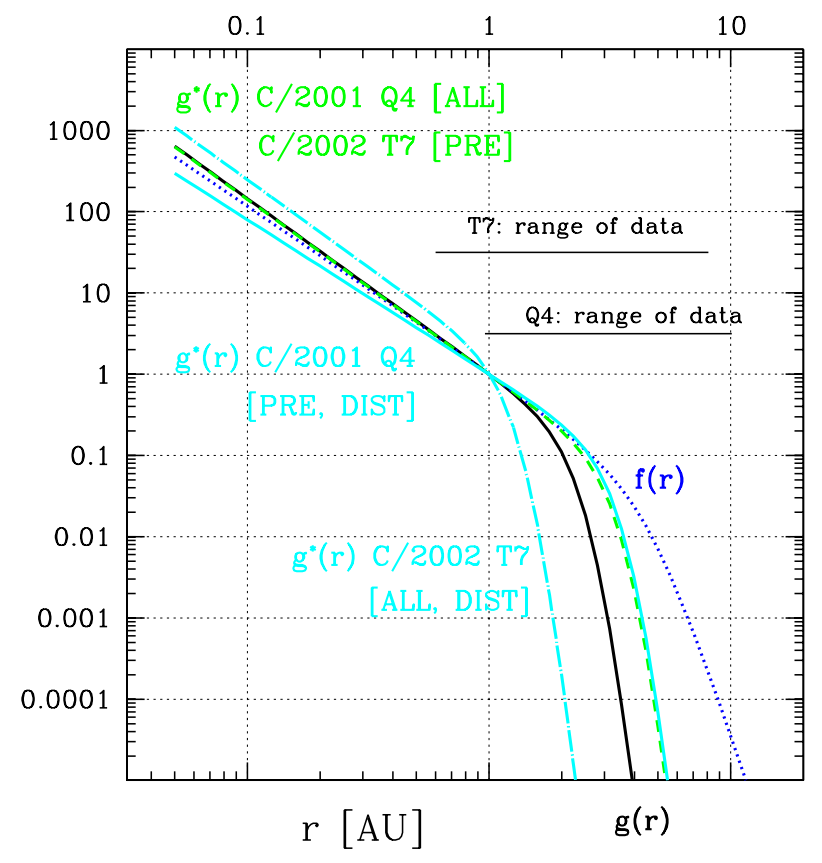

Fig. 3. Different forms of the NG function $h(r)$ versus (vs.) heliocentric distance $r$ (Eqs. (3)-(5)) investigated in this paper. Standard $g(r)$ is shown with a black curve, and a Yabushita function $f(r)$ with a dotted blue (dark grey) one. From top to bottom, according to description given in the figure: dashed green/grey $\left(m=-2.15, r_{0}=4.0\right)$, solid cyan/grey $\left(m=-1.9, r_{0}=4.0\right)$, and dotted-dashed cyan/grey ( $m=-2.15, r_{0}=1.5$ ) curves represent the best derived $g(r)$-like functions for the comets C/2001 Q4 and C/2002 T7 for three types of data (ALL, PRE, and DIST, see Sect. 3.1).

of data seem to be more adequate for the determination of the starting osculating orbit for previous perihelion dynamical calculations than models based on the whole data set. Observations from PRE data subsets cover significantly shorter time intervals and the osculating orbits derived on their basis should be treated with greater caution than orbits based on DIST data subsets. However, in some situations, for example when a comet splitting event occurs, such a model might be the only appropriate.

\subsection{Models of non-gravitational motion}

The classical method of NG orbit determination was originally proposed by Marsden et al. (1973). Their widely used model assumes that accelerations are symmetric with respect to the perihelion and is expressed by the semi-empirical function $g(r)$

$$
\begin{array}{r}
F_{i}=A_{i} \cdot g(r), \quad A_{\mathrm{i}}=\text { const. for } i=1,2,3, \\
g(r)=\alpha\left(r / r_{0}\right)^{-2.15}\left[1+\left(r / r_{0}\right)^{5.093}\right]^{-4.6142},
\end{array}
$$

where $F_{1}, F_{2}$ and $F_{3}$ are the radial, transverse, and normal components of the NG acceleration, respectively, $\alpha=0.1113$ is a normalization constant defined by the relation $g(1 \mathrm{AU})=1$, and the scale distance $r_{0}=2.808 \mathrm{AU}$ is a heliocentric distance where solar radiation triggers the effective sublimation of water ices. The standard form of the dimensionless function $g(r)$ is related to an empirical water-ice sublimation curve derived by Delsemme \& Miller (1971). Thus, at small heliocentric distances, $r \ll r_{0}$, NG force is roughly proportional to $r^{-2.15}$, whereas at $r \gg r_{0}$ it drops faster with distance from the Sun, as modelled by the second term in the formula for the $g(r)$-function (see black curve in Fig. 3).
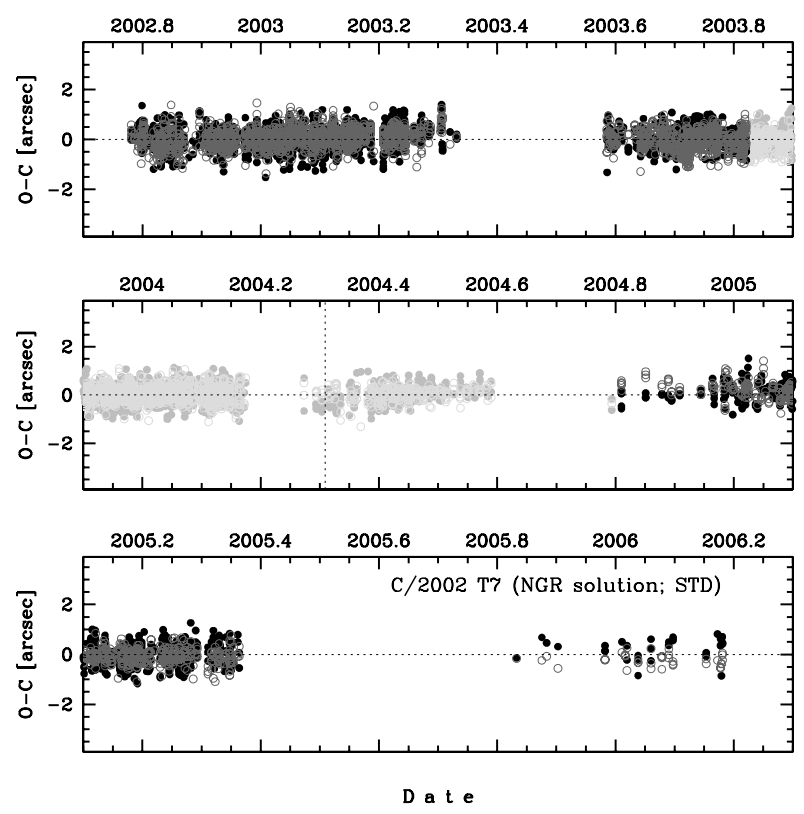

Fig. 4. The O-C diagram for comet C/2002 T7 LINEAR for two separate NG models of a standard acceleration derived for two complementary data sets. Residuals for the NG model based on the DIST data sets (observations taken when the comet was further than 3.0 AU from the Sun, see Sect. 3.1) are shown by black dots and dark grey circles (residuals in right ascension and declination, respectively). The other NG model was derived for the remaining data (taken when the comet was closer than 3.0 AU from the Sun) and residuals are shown in light grey; the moment of perihelion passage is shown by a dashed vertical line.

This standard form of $g(r)$ was originally derived for shortperiod comets. However, it is also often used to determine $\mathrm{NG}$ effects in the motion of long-period comets since the relevant function for the family of LP comets has not yet been found. Moreover, it seems that LP comets differ significantly from each other in terms of the sublimation rate variability as a function of heliocentric distance; the comets C/1995 Q1 Hale-Bopp and C/1996 B2 Hyakutake may serve as good examples of this phenomenon (Szutowicz et al. 2002b,a).

Thus, in the present investigation, instead of a standard $g(r)$ function described by Eq. (1) we introduce a more general form of the dependence of NG acceleration on the heliocentric distance

$F_{i}=A_{i}^{*} \cdot h(r), \quad A_{i}^{*}=$ const. for $i=1,2,3$,

and we adopt three different forms for a dependence of acceleration on the heliocentric distance, $h(r)$, namely standard $g(r)$ based on water sublimation, more general $g(r)$-like function $g^{*}(r)$, and Yabushita function, $f(r)$, based on the carbonmonoxide sublimation rate (Yabushita 1996). Thus, we consider here the following three types of NG models:

- STD NG model type

$h(r)=g(r)$;

- GEN NG model type

$h(r)=g^{*}(r)=\alpha\left(r / r_{0}\right)^{-m}\left[1+\left(r / r_{0}\right)^{n}\right]^{-k} ;$

- YAB NG model type

$h(r)=f(r)=\frac{1.0006}{r^{2}} \times 10^{-0.07395(r-1)}\left(1+0.0006 r^{5}\right)^{-1}$. 
In the GEN type of NG model, we have an additional four free parameters: scale distance, $r_{0}$, and the exponents $m, n$ and $k$. The function $g^{*}(r)$ is normalized similarly as a standard $g(r)$ function, thus $\alpha$ is calculated from the condition $g^{*}(1 \mathrm{AU})=1$, and the $f(r)$ function is normalized to unity at $r=1 \mathrm{AU}$ (see dotted curve in Fig. 3).

In the above three types of NG models, all three NG parameters $A_{1}^{*}, A_{2}^{*}$, and $A_{3}^{*}$ (Eq. (2)) were derived together with six Keplerian orbital elements in the iterative process of the orbital determination within a given observational time-interval defined by the type of the data set (ALL, DIST, PRE, see Sect. 3.1). In the GEN type of NG models, we additionally examined the various values of scale distance, $r_{0}$, and exponents $m, n$ and $k$. Unfortunately, the dependence of the $g^{*}(r)$-formulae on the particular choice of astrometric data is weak. For both comets, the orbit fitting is most sensitive to changes in the scale distance $r_{0}$ and to a lesser extent changes in the value of an exponent $m$. Thus, in our subsequent analysis we kept the standard values of the exponents to $n=5.093$ and $k=-4.6142$. However, according to our modelling the $g(r)$-like formula should decline more steeply at large than at small heliocentric distances. Thus, the $r_{0}$ seems to be important to the modelling of the NG forces in both comets. Omitting completely the $r_{0}$ parameter as a turning point in the $g(r)$-like behaviour (Eq. (4)), we obtain worse fits.

Since the detection of NG effects in astrometric data of LP comets is rather difficult, we tried to model NG accelerations with a number of NG parameters no larger than necessary to improve the model fit to the data. For example, we tested additionally the asymmetric NG model, which introduces an additional NG parameter $\tau$, namely the time displacement of the maximum of $h(r(t-\tau))$ relative to the moment of perihelion passage. According to our calculations based on ALL type of data, the asymmetric standard model of NG motion of C/2001 Q4 gives only a slight shift of $0.85 \pm 0.45$ day after the moment of perihelion passage. However, this model gives: (i) the same rms as the symmetric model, (ii) similar trends in the $\mathrm{O}-\mathrm{C}$ diagrams for orbit fitting, and (iii) a similar deviation for the $\mathrm{O}-\mathrm{C}$ distribution from normal distribution. We tested an asymmetric model for both comets and all types of data subsets and noticed no improvement in any of the above three elements relative to the corresponding symmetric models. Thus, we present in this paper only symmetric models.

\section{New results}

\subsection{Previous perihelion distance calculations}

After obtaining for both comets original (before entering our planetary system) and future (when leaving our planetary region) orbits, we calculated their orbits at a previous perihelion passage (both comets leave the solar system on hyperbolic orbits, so their future dynamics is clear). We used here the same methods and algorithms as applied in Paper I. As early as at the moment of osculation (i.e. close to the perihelion passage), the single cometary orbit was replaced by a swarm of 5000 randomly chosen (but representative of the observations) VC orbits, and all of them were propagated to both the past and future, giving us swarms of original and future orbits. To obtain as much information about the previous perihelion passage of two comets investigated in this paper as possible, we followed numerically all VC orbits in these swarms back to the previous perihelion; all details of the numerical technique used here can be found in Paper I, and some additional information can be found in Dybczyński \& Królikowska (2011). This was done for

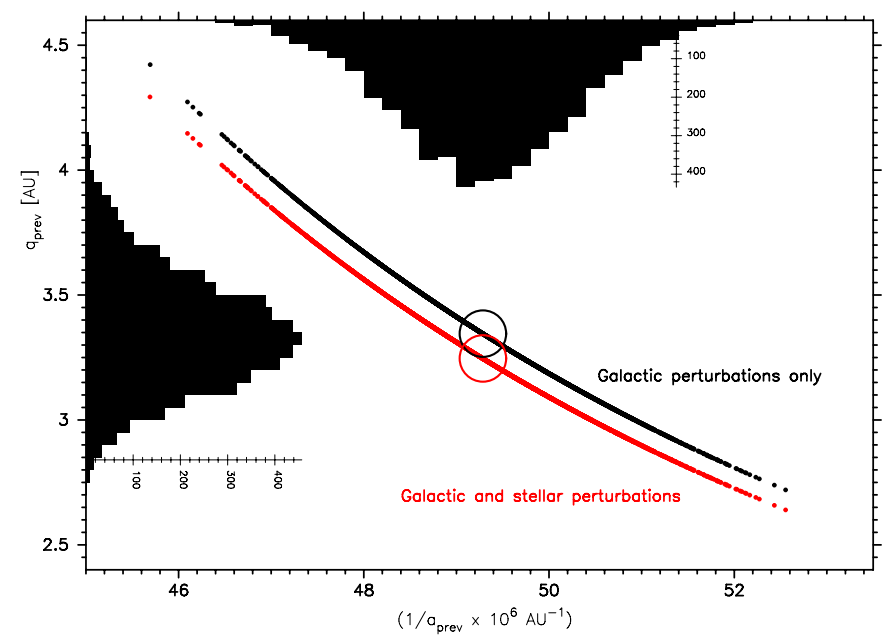

Fig. 5. The dependence of the previous perihelion distance on the reciprocal of the previous semimajor axis for C/2001 Q4 (DIST2 variant with the standard NG model). Each dot in the central part of figure represents an individual cometary orbit from the swarm of $5000 \mathrm{VC}$ orbits propagated to the previous perihelion ( $\sim 3$ million years back in time). Results of two different calculations are shown: without stellar perturbations (the central upper plot) and with stellar perturbation included (the lower one). The centres of the big circles define the positions of the respective nominal orbits. For the variant without stellar perturbations, boundary distributions of $1 / a_{\text {prev }}$ and $q_{\text {prev }}$ are also plotted.

all variants of NG force models investigated here, as well as for all types of the observational-material sub-samples.

The detailed example of our results for the previous perihelion of C/2001 Q4 (DIST2 type of data set and standard NG force model) is depicted in Fig. 5. This picture also shows the boundary VC distributions of the previous perihelion distance, $q_{\text {prev }}$, and the reciprocal of the previous semimajor axis, $1 / a_{\text {prev }}$. It is noteworthy, that owing to the nature of the Galactic disk perturbations (dominating here), the semimajor axis remains almost unperturbed so its distribution is almost exactly the same as that of $1 / a_{\text {ori }}$. It is easy to note that both distributions, $q_{\text {prev }}$ as well as $1 / a_{\text {prev }}$, are fairly Gaussian.

During the past dynamical evolution, we took into account tidal perturbations from both the Galactic disk and the Galactic centre. Additionally, we checked the potential perturbing-effect of all known stars that had passed close to the solar system during the past ten-million years. Dybczyński (2006) showed that there had been no significant change in the cometary orbital elements at the previous perihelion passage after including known stellar perturbations, but recently new data on potential stellar perturbers were made available. The complete new reduction of the Hipparcos satellite measurements were performed and the new version of the catalogue (HIP2) was made available (see van Leeuwen 2007, for details). Using this new set of precise stellar data, Anderson \& Francis (2012) prepared a compilation of them with other stellar characteristics, including radial velocities. On this basis, they searched for any stars that had visited the solar system neighbourhood, by calculating their minimal heliocentric distances and epochs of the closest proximity. We therefore extended our dynamical model including gravitational perturbations from 35 stellar systems. This set of stellar perturbers were obtained by combining the results of Dybczyński (2006) and Anderson \& Francis (2012). Again, no significant perturbation from the passing stars was detected.

Thus, the dominating perturbing forces in the motion of both comets at huge heliocentric distances are the tidal gravitational 
actions of the Galactic disk and Galactic centre. The combination of the Galactic tides and planetary perturbations constitute fundamentals of a well-known concept called the Jupiter-Saturn barrier. We discussed this in detail in Dybczyński \& Królikowska (2011), where we also presented several examples of large perihelion-distance comets that traverse this barrier, sometimes without any significant orbit changes. From the Jupiter-Saturn barrier point of view, long-period comets can be divided into two dynamical classes: jumpers and creepers ${ }^{2}$. When Galactic tides reduce the perihelion distance of a particular comet from above say $15 \mathrm{AU}$ down to the observable region in one orbital period, we call such a comet a jumper since it jumps over the Jupiter-Saturn barrier. Otherwise, if the perihelion distance evolution under the Galactic perturbations is much slower, we call such a comet a creeper, since it is passes several times through the strong planetary perturbations zone, fortunately not experiencing significant orbit change.

Among the two comets investigated in this paper, C/2001 Q4 NEAT is a typical example of a creeper, while C/2002 T7 LINEAR seems to be a typical jumper. This distinction is based on a previous perihelion distance of these comets, and results in this case (and most other cases) mainly from the different values of the semimajor axes. This dependence of previous perihelion distance on semimajor axis is obvious only in the absence of any significant stellar perturbations.

While for $\mathrm{C} / 2002 \mathrm{~T} 7$, the relative effect of stellar perturbations is really infinitesimal, in the case of C/2001 Q4 it is still very small but more apparent, as shown in Fig. 5. It is easy to note a systematic shift to smaller perihelion distances (about $0.10-0.15$ AU, i.e. about 3 per cent) after including stellar perturbations (red dots), while the $1 / a_{\text {prev }}$ values do not change. It should be stressed here that the previous perihelion passage of C/2001 Q4 occurred only 2-4 million years ago (depending on the NG force and type of data model used) so our knowledge of the potential stellar perturbers of its motion seems to be fairly complete. The small difference presented in Fig. 5 is quite representative of the whole grids of models analysed here. Thus, we are convinced that known stars do not influence our conclusions on the dynamical history of both investigated comets by any means.

The overall dependence of the previous perihelion distance on the original semimajor axis for all these variants is depicted in Fig. 7, and our numerical results are summarized in Tables 3 and 4.

In Fig. 6, we show the backward dynamical evolution of VC swarms of C/2001 Q4 and C/2002 T7 where two swarms for each comet are shown. The main source of differences in the past evolution are the differences in the original semimajor axes. It is also clearly visible that swarms based on subsets of data (PRE and DIST2 models) are more dispersed than the respective swarms based on the entire set of observational material (ALL models). For this comparison, we used the standard form of the NG forces in each case and switched off our "escape threshold" taken at 120000 AU to allow for all VCs to reach the previous perihelion.

\section{2. $C / 2001$ Q4 NEAT}

According to Kammerer (2011), the brightness variation of comet NEAT with heliocentric distance shows two main phases

\footnotetext{
2 These terms were probably first used in this context by Hans Rickman in his review of cometary dynamics (Rickman 2010).
}

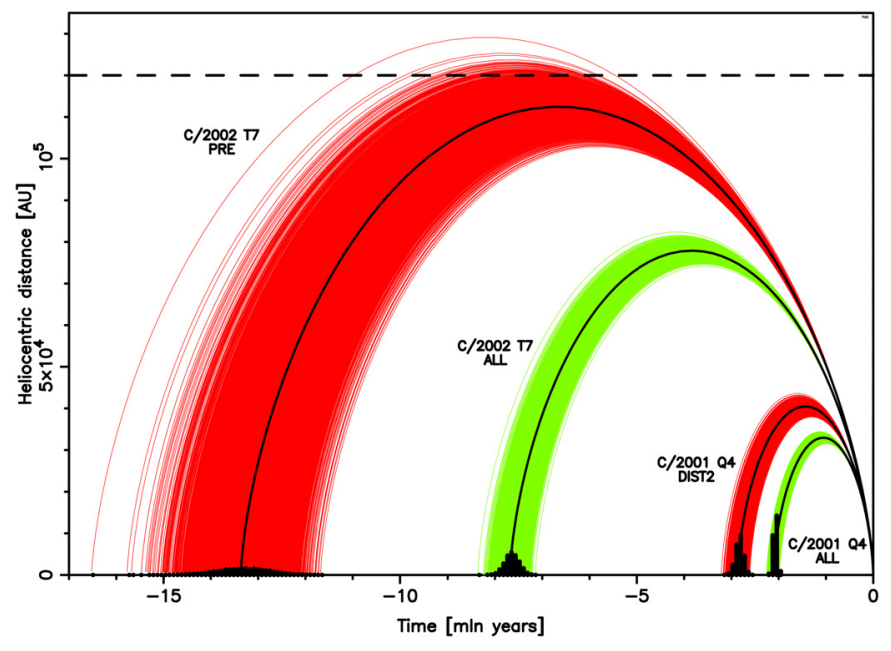

Fig. 6. The comparison of the backward dynamical evolution of the VC swarms of C/2002 T7 and C/2001 Q4. For each comet, two different swarms are shown: a green (light grey) based on all astrometric data and a red (dark grey) representing our preferred data model for a particular comet (PRE for C/2002 T7 and DIST2 for C/2001 Q4). In each case, the nominal orbit is drawn in black. We show also in black, the histograms of the swarm density at the moment of a previous perihelion passage. The horizontal dashed line depicts the "escape limit" of $120000 \mathrm{AU}$ used in our investigations.

in pre- and post-perihelion data, separately. He derived powerlaw fits for four periods, where the borders were set at 90 days before perihelion passage (at 1.6 AU from the Sun), at the moment of perihelion, and 85 days after perihelion passage (also at about 1.6 AU from the Sun). The average brightness evolution with heliocentric distance was significantly faster when this comet was further than 1.6 AU from the Sun along the incoming leg of the orbit, as well as along the outgoing leg. Around perihelion, when the comet was closer than 1.6 AU from the Sun, the average variations in the brightness seemed to be smaller, however several local maxima and minima are visible in the light curve, for example Kammerer points to a significant minimum just before perihelion (between 20 April and 5 May) and then a sharp increase until perihelion. According to Churyumov et al. (2008), the power-law slope of the brightness variation with heliocentric distance changed four times before perihelion and four times after. They also concluded that these photometric changes occurred at very similar heliocentric distances before and after perihelion. However, they pointed out that the brightness variation was generally steeper before the perihelion passage than after.

Similar conclusions were derived from the water-production rate curve. The hydrogen Ly $\alpha$ comae of both comets was detected by the SWAN all-sky camera on the SOHO spacecraft. The comet C/2001 Q4 NEAT was observed (Combi et al. 2009) from the heliocentric distance of $3.23 \mathrm{AU}$ before to $2.75 \mathrm{AU}$ after perihelion (14 September 2003-2 November 2004). They found that the average water-production rate varies with heliocentric distance as $r^{-1.7}$. However, they concluded that this comet was definitely more active before than after perihelion and that power-law fitting gives significantly different powerlaw slopes for pre- and post-perihelion changes in the water production rate with the heliocentric distance $r$. They found that the best-fit power-law variations in the water production rate of $\mathrm{C} / 2001 \mathrm{Q} 4$ are proportional to $r^{-2.2}$ and $r^{-1.7}$ for the pre- and post-perihelion data, separately. This means that a power-law function of water production rate generally seems to be steeper 
before perihelion passage than after, which is in agreement with Churyumov et al. (2008). However, Combi et al. (2009) reported that comet NEAT "showed a production rate outburst about 30 days before perihelion (15 April 2004) and then a large extended increase above the nominal trend from 50 to 70 days after perihelion (2004 July 5-July 25)". In their paper, Fig. 2 shows that a power-law function with one slope is a very coarse approximation of actual water production behaviour before perihelion.

To avoid problems when modelling local anomalies in the NG accelerations as a function of heliocentric distance, we constructed three sets of data for this comet based on the analysis of a water production rate curve described by Combi et al. (2009). In the DIST2 type of data, we ejected the smallest possible number of observations around perihelion, namely from 15 April 2004 to 25 July 2004, i.e. 1163 observations (44\% of all data) taken from 1.2 $\mathrm{AU}$ before perihelion passage to $1.5 \mathrm{AU}$ after perihelion passage. In addition, we finished the PRE type subset of data at 15 April 2004. The third type of data subset (DIST1) includes only observations taken at large distances from the Sun (further than 3.0 AU). This means that in the case of comet C/2001 Q4 NEAT, we ejected all observations around perihelion taken closer than 3.0 AU from the Sun, i.e. from 9 November 2003 to 23 November 2004 (74\% of all observations). Thus, the DIST1-subset of data covers the period almost entirely outside the SWAN observations. More details about the observational materials are given in Table 3 .

In the case of the GEN type of NG motion, we noticed that there is a decrease in rms for $r_{0}$ greater than the standard value of 2.808 AU and smaller than 6.0 AU. As the most representative value, we took $r_{0}=4.0 \mathrm{AU}$. The full set of observational material (ALL type of data set) seems to be insensitive to the $m$ exponent, thus we assumed the standard value of $m=-2.15 \mathrm{AU}$ (Col. 3 of Table 3 and dashed green/grey curve in Fig. 3). For the remaining type of data (PRE, DIST1, and DIST2), the closest data fitting were derived for $r_{0}=4.0 \mathrm{AU}$ and $m=-1.9$ (Cols. 4-6 of Table 3 and solid cyan/grey curve in Fig. 3). The slower $h(r)$ change with heliocentric distance than standard $\left(m_{\mathrm{STD}}=-2.15\right)$ is a very interesting result and agrees with Combi et al. (2009).

For all these starting models ( 4 types of data $\times 3$ types of NG motion), we finally derived the original semimajor axes, $1 / a_{\text {ori }}$ taken at $250 \mathrm{AU}$ from the Sun in the case of backward dynamical evolution, and the values of previous perihelion distances, $q_{\text {prev }}$. Results are presented in Table 3 and Fig. 7. All black parts of the NEAT curve show ranges of $1 / a_{\text {ori }}$ and $q_{\text {prev }}$ determined for four investigated types of data sets (from top to bottom: PRE, DIST1, DIST2, ALL). Within the given type of data set, ranges for three types of NG models overlap or partly overlap (as for DIST1 and PRE data sets), or are separated from each other (as for the DIST2 and ALL data sets), where the order of NG models depends on the type of data set.

As described in detail above, from the PRE, DIST1, and DIST2 data sets, we excluded periods where local anomalies in the cometary activity occurred (Combi et al. 2009), thus for our purposes models based on these three subsets should be more reliable than models based on all the observations (ALL type of data). On the other hand, the lack of reports of cometary break-up (or large outbursts outside the excluded period from 15 April 2004 to 25 July 2004) allows us to conclude that models based on the richer and longer data sequences should be preferred over models based on shorter data sequences. Thus, in the case of comet C/2001 Q4 we can recommend models based on the DIST2 type of data.

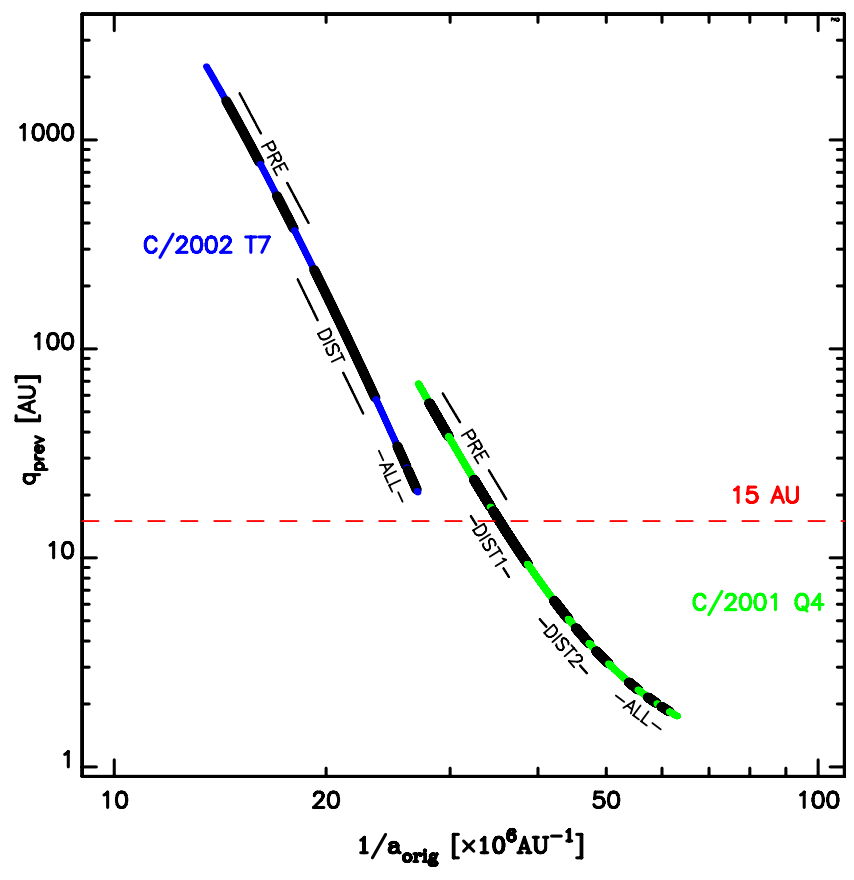

Fig. 7. Relations of previous perihelion distance vs original semimajor axis for C/2001 Q4 NEAT (green curve) and C/2002 T7 LINEAR (blue curve). The black parts of NEAT curve show ranges of values determined in four investigated type of data sets (PRE, DIST1, DIST2, ALL) and black parts of LINEAR curve show the ranges of values derived in three types of data sets (PRE, DIST and ALL).

\subsection{C/2002 T7 LINEAR}

The comet LINEAR was observed by SWAN from 2.52 AU before to 2.09 AU after perihelion (4 December 2003-6 August 2004). Combi et al. (2009) reported for this comet that no large outburst was detected during the observed period. They found that the average water-production rate varies with heliocentric distance as $r^{-2.0}$, i.e. is steeper than for $\mathrm{C} / 2001 \mathrm{Q} 4$. The best-fit power-law approximation to the water production variations of $\mathrm{C} / 2002 \mathrm{~T} 7$ is proportional to $r^{-1.6}$ and $r^{-2.1}$ for pre- and post-perihelion measurements, respectively. This means that the power-law function of water production rate here is flatter before perihelion than after, e.g. unlike in the case of C/2001 Q4, although both comets were generally more active before than after perihelion.

It was reported, however, that a significant change in the brightness trend occurred before the SWAN campaign, namely around 25 October 2003 when a comet was about 3.0 AU from the Sun (Kammerer 2011). According to Kammerer (2011), the second break in the comet's brightness evolution took place at the beginning of February 2004 (about 1.6 AU from the Sun). Discussing the anti-tail behaviour, Sekanina (2004) found that the dust that formed this anti-tail had been ejected most probably in the period between discovery and the end of June 2003, e.g. in the distance greater than 4.5 AU from the Sun and about one year before perihelion passage or even before discovery. In this situation, the independent investigation of NG motions based on different subsets of data could give us some new information.

For the ALL and DIST types of data sets, we found similar trends in the NG effects, namely, in the case of the GEN type of NG motion, we noticed a decrease in rms for $r_{0}$ significantly smaller than its standard value and used $r_{0}=1.5 \mathrm{AU}$ (dotted-dashed cyan/grey curve in Fig. 3). However, for the preperihelion type of data (PRE) the best data fitting was derived 
for $r_{0}=4.0 \mathrm{AU}$, as in the case of the comet $\mathrm{C} / 2001 \mathrm{Q} 4$ according to the last column of Table 4 and dashed green/grey curve in Fig. 3. Unfortunately, all the types of data sets seems to be insensitive to the $m$ exponent around its standard value, thus we assume the standard value of $m=-2.15 \mathrm{AU}$.

For all these starting models ( 3 types of data $\times 3$ types of NG motion), we finally found that the original, barycentric semimajor axis taken at $250 \mathrm{AU}$ from the Sun in backward dynamical evolution is in the range of $14<1 / a_{\text {ori }}<27$ in units of $10^{-6} \mathrm{AU}^{-1}$. At first glance, this range appears to be narrow. However, it results in a wide range of previous perihelion distances from $23 \mathrm{AU}$ to $403 \mathrm{AU}$ for returning orbits. Moreover, for PRE type of data, and $g(r)$-like or Yabushita types of NG motion, full swarms of VC orbits are escaping. It is worth explaining here our definitions of returning and escaping orbits. When numerically following each VC motion, we limited ourselves to the heliocentric distance of 120000 AU. Orbits with an aphelion distance smaller than that limit are call returning, the remaining orbits from the swarm are called escaping. However, the majority of the escaping orbits are still elliptical. Since we deal with swarms of 5000 individual orbits of VCs instead of the single, nominal orbit, we found in some cases that part of the swarm is returning, while the rest is escaping and probably that some of the escaping VCs have hyperbolic orbits. The details of our numerical procedure and the methods of result interpretation are described in detail in Paper I.

For the two comets investigated in this paper, we found that all investigated swarms of VC orbits for C/2001 Q4 consist of returning orbits. This is the same situation we found in the case of C/2002 T7, except all three NG models based on the PRE sample of observations. For the standard NG model (STD), we obtained 61 escaping orbits (see Fig. 6), for the generalized NG model (GEN) we found 4980 escaping orbits, while using the Yabushita function (YAB model) all orbits in the swarm were escaping. It is noteworthy to mention that all these escaping orbits are fairly elliptical, all aphelion distances are smaller than $125000 \mathrm{AU}$ for STD model, smaller than $135000 \mathrm{AU}$ for GEN model, and smaller than $150000 \mathrm{AU}$ for the YAB model. It might be added for completeness that all swarms of future orbits for both comets only consist of hyperbolic orbits.

The black parts of the comet $\mathrm{C} / 2002 \mathrm{~T} 7$ curve in Fig. 7 show ranges of $1 / a_{\text {ori }}$ and $q_{\text {prev }}$ determined in three investigated type of data sets (from top to bottom, PRE, DIST, and ALL). As in the case of comet $\mathrm{C} / 2001 \mathrm{Q} 4$, ranges for different types of NG models overlap within the individual type of data set (as for DIST and ALL data sets) or are sometimes separated (as for the PRE data set). In the case of C/2002 T7, there is no doubt that this comet is dynamically new.

There are also reports about the possibility of the nucleus fragmentation of this comet (Milam et al. 2006). Hence, the best way to determine the unadulterated values of $1 / a_{\text {ori }}$ and $q_{\text {prev }}$ is probably to take into account only observations before perihelion to determine the starting orbit for dynamical studies on the previous perihelion passages. On the basis of PRE type of data, we conclude that the previous perihelion was far beyond the planetary system, where the lowest value of $q_{\text {prev }} \simeq 400 \pm 80 \mathrm{AU}$ was obtained for the STD type of NG models.

\subsection{Differences in dynamical evolution under the Galactic tides}

As concerns the dynamical evolution under the Galactic perturbations, we can note several differences and more similarities

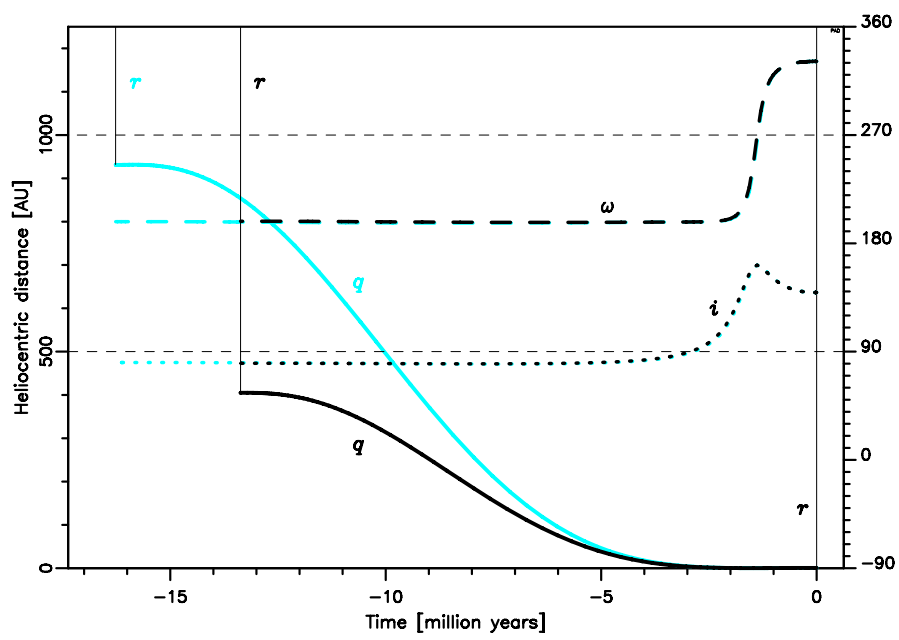

Fig. 8. Backward dynamical evolution of C/2002 T7 for a nominal VC from the PRE+GEN swarm (cyan/grey) and PRE+STD swarm (in black). The evolution of the perihelion distance (thick solid line) and the heliocentric distance (thin solid lines) are shown with the left vertical axis in AU. The right vertical axis in degrees corresponds to the plots of the argument of perihelion (thick dashed line) and the inclination (dotted line), both in the Galactic reference frame.

between the two comets investigated here. C/2001 Q4 was observed several million years before the secular minimum of its perihelion distance, during the decreasing phase of this element evolution with the argument of perihelion slowly increasing in the first quarter (this comet has a prograde orbit in the Galactic frame).

In contrast, as depicted in Fig. 8, C/2002 T7 was observed just after the secular minimum of the perihelion distance evolution (the observed value of an argument of perihelion in the fourth quarter) and its orbit is retrograde in the Galactic frame. In this figure, we present two different dynamical evolutions: the nominal VC of the PRE+STD model (in black) is superimposed on the evolution of the orbital elements of the nominal VC from the PRE+GEN swarm (in cyan/grey). It is clearly shown that the only significant difference (i.e. where grey lines are visible) is found for the evolution of the perihelion distance (thick solid lines). This is of course the result of the different semimajor axes obtained in these two models - the difference in the orbital period is also depicted. Owing to the influence of the Galactic centre perturbation, this comet switched from a prograde to a retrograde orbit $\sim 3$ million years ago and since that moment its Galactic inclination ( marked with a dotted line) has increased from 90 degrees up to almost $\sim 170$ degrees and decreased again to $\sim 140$ degrees before the observed perihelion passage. This behaviour of the inclination evolution would be impossible under the separate Galactic disk tide so we have here a nice example of the significant qualitative influence of the tidal perturbation originating from the Galactic centre. The evolutions of the argument of perihelion (the dashed line) and the heliocentric distance (thin vertical lines) are also shown.

Though, the main difference between the past dynamical evolutions of these comets is their significantly different values of original semimajor axes (depending on the model: 16400-35 $700 \mathrm{AU}$ for $\mathrm{C} / 2001 \mathrm{Q} 4$, and 38 500-67 000 AU for $\mathrm{C} / 2002 \mathrm{~T} 7$ ), there is a systematic trend in $a_{\text {ori }}$-changes for investigated comets: the smallest semimajor axis for both comets results from the ALL type of data set, a larger one from the DIST-type of data, and the largest from the PRE-type of data. As a result, the previous perihelion distance was, respectively, 
moderately, more, and largely increased (going to the past) by Galactic perturbation for both comets. Because of the larger semimajor axis, C/2002 T7 is much more sensitive to even small differences in the original orbit. For example, updating the observational material described in Table 1, we noticed several additional observations in February 2004 (compared to data taken from the earlier database of Paper I), i.e. two months before the end of PRE data set. This small difference in the number of observations results in a change in the nominal, previous perihelion distance for the PRE-type data set from 274 AU to $405 \mathrm{AU}$. This is consistent however with the combined standard error obtained for these two solutions.

\section{Summary and conclusions}

The purpose of the present research was not only that raised in the question of the title of this paper but was also to show how different model assumptions affect the past dynamical evolution of so-called Oort spike comets $\left(1 / a_{\text {ori }}<10^{-4} \mathrm{AU}^{-1}\right)$ with osculating orbits classified with the highest accuracy. To achieve this, we have examined the past evolution of both $\mathrm{C} / 2001 \mathrm{Q} 4$ and $\mathrm{C} / 2002 \mathrm{~T} 7$ for three types of data series. We have constructed data series taken before perihelion passage (PRE type of data), as well as a data set composed of observations taken at large distances from the Sun (DIST type of data) based on the water production-rate behaviour of these comets. Although we have presented complete grids of models for all three types of data sets, we have argued that in the case of comet C/2002 T7, models based on pre-perihelion data (PRE type of data set, see Table 4) seem to be the most useful for determining the previous perihelion distance, while in the case of comet C/2001 Q4 the best are DIST-type models, in particular the DIST2 version (see Table 3).

However, the analysis of the complete grid of models has also been very instructive. It shows that the greatest change in the previous perihelion relative to that obtained in the canonical approach (a complete set of astrometric data and the standard $g(r)$-form of NG effects) results from the different types of data subsets used for each of these two comets. In this context, the form of the NG acceleration dependence on the heliocentric distance is of secondary importance (see Fig. 7).

Astrometric data of the investigated two comets have allowed to identify a clear existence of dynamic effects associated with the NG effects. We have therefore tried to examine whether it is possible to decide which variant of the NG acceleration dependence on the heliocentric distance described by Eqs. (3)-(5) most closely fits the data. We have found that for all three types of data sets, the standard $g(r)$-function seems to fit the data worse than the $g(r)$-like function and sometimes worse than the $f(r)$-function given by Yabushita. The only exception was the models based on all observations (ALL data type) for the comet C/2001 Q4 where we did not observe any improvement in the rms (as well as the $\mathrm{O}-\mathrm{C}$ distribution and $\mathrm{O}-\mathrm{C}$ diagrams) for either the $g(r)$-like function or $f(r)$-function compared to the standard $g(r)$-function. The Yabushita function never was able to fit any of the data better than other forms of NG acceleration so the results of the YAB model based on a CO-sublimation are presented here mainly for completeness and comparison purposes. The astrometric data shows that the best form of NG acceleration seems to be the $g(r)$-like function, which has a substantially different $r_{0}$ from that given in the standard $g(r)$ function. Thus, we concluded that the NG acceleration in both comets do not follow the theoretical $g(r)$-function expected for an isothermal nucleus. Additionally, in the PRE type of data of C/2002 T7, the derived $g(r)$-like function seems to be closer to a model of outgassing restricted to the subsolar point on the nucleus surface originally introduced by Sekanina (1988) and defined by the parameters: $r_{0}=5.6, m=2.1, n=3.2$, and $k=3.9$.

In the case of comet C/2001 Q4, for three types of data subsets (PRE, DIST1, and DIST2) we found that the best-fit models are based on $g(r)$-like function with $r_{0}=4.0 \mathrm{AU}$ that are proportional to $r^{-1.9}$, i.e. have a distance scale larger than the standard $r_{0} \simeq 2.8 \mathrm{AU}$ and a weaker dependence on heliocentric distances than the standard $\sim r^{-2.15}$ (at small distances from the Sun). The latter result is qualitatively consistent with the results of Combi et al. (2009), who included this comet in the water variation group with shallow slopes (Table 5 in their paper). Using DIST2 data, constructed on the basis of Combi et al. (2009) conclusions about increase activity of C/2001 Q4 above the nominal trend around perihelion (from 30 days before perihelion to 70 days after), we found that this comet was well inside the Saturn orbit during a previous perihelion passage $(\sim 2-4$ million years ago).

A similar analysis of a grid of models for comet C/2002 T7 also showed that models based on a $g(r)$-like function provided a closer fit to the data than standard models. However, the Yabushita function was as good as the $g(r)$-like function for the PRE data set. Unfortunately, our conclusion about the form of a $g(r)$-like function is much less clear than in the case of comet C/2001 Q4, because depending on the data type we obtain a different value of the scale distance $r_{0}$ (see Table 4). We were also unable to identify any qualitative difference in the fitting of models with a standard slope $m=-2.15$ and either smaller or larger values of an exponent in the range $m=-2.15 \pm 0.15$, i.e. a steeper slope than -2.3 or one flatter than -2.0 already gave a noticeably poorer fit. This result is also qualitatively consistent with Combi et al. (2009), who - according to the productionrate power-law slope classification of Combi et al. (2008) - include C/2002 T7 in the moderate slope group. However, all models tested here indicate that this comet was outside the orbit of Uranus in the previous perihelion passage $\sim 8-14$ million years ago. Since there are reports of nucleus fragmentation, we believe that the safest approach for C/2002 T7 is to apply the PRE type of data set and then draw conclusions about its past evolution. Models based on this set of data indicate that the comet was far outside the planetary zone during the previous perihelion passage $\left(q_{\text {prev }} \gg 50 \mathrm{AU}\right)$.

More details and plots for both comets studied in this paper will successively appear at our project web page ${ }^{3}$.

Among the comets with a small perihelion distance $(q<$ 3.0 AU) and original semimajor axis $1 / a_{\text {ori }}<10^{-4} \mathrm{AU}^{-1}$ discovered so far, only a few have positional material rich enough to determine the form of the dependence of the NG acceleration on the heliocentric distance. In the future, we will extend our study of the past dynamical evolution of comets to those of the recently discovered objects with small perihelion distances and $1 / a_{\text {ori }}<10^{-4} \mathrm{AU}^{-1}(\sim 20$ comets in the past ten years $)$, and the analysis presented here will be performed for all objects discovered far enough away from the Sun.

Answering the title question, we can conclude that C/2001 Q4 is not a dynamically new comet and that it visited the planetary perturbation zone at least once during its previous perihelion passages. We call such a comet a creeper. Conversely, C/2002 T7 seems to be a good example of a jumper, i.e. comet experiencing large Galactic perturbations that greatly reduced its perihelion distance during its last orbital revolution, hence did

\footnotetext{
3 Dybczyński \& Królikowska in http://apollo.astro . amu . edu .pl/WCP
} 
not suffer any planetary perturbations at the previous perihelion passage.

Acknowledgements. The research described here was partially supported by the Polish Ministry of Science and Higher Education funds (181/N-HSO/2008/0). The authors are very grateful to the referee, Ramon Brasser, for his very valuable comments, which allowed us to improve the paper.

\section{References}

Anderson, E., \& Francis, C. 2012, Astron. Lett., 38, 331

Buffington, A., Bisi, M. M., Clover, J. M., et al. 2008, ApJ, 677, 798

Churyumov, K. I., Filonenko, V. S., \& Chubko, L. S. 2008, Kinemat. Phys. Celest. Bodies, 24, 105

Combi, M. R., Mäkinen, J. T. T., Henry, N. J., Bertaux, J.-L., \& Quemérais, E. 2008, AJ, 135, 1533

Combi, M. R., Mäkinen, J. T. T., Bertaux, J.-L., Lee, Y., \& Quémerais, E. 2009, AJ, 137, 4734

Delsemme, A. H., \& Miller, D. C. 1971, Planet. Space Sci., 19, 1259

Dybczyński, P. A. 2006, A\&A, 449, 1233

Dybczyński, P. A., \& Królikowska, M. 2011, MNRAS, 416, 51
Fernández, J. A., \& Sosa, A. 2012, MNRAS, 423, 1674

Gibb, E. L., Bonev, B. P., Mumma, M. M., et al. 2007, in BAAS 38, AAS/Division for Planetary Sciences Meeting Abstracts, 39, 522

Hogerheijde, M. R., Qi, C., de Pater, I., et al. 2009, AJ, 137, 4837

Kammerer, A. 2011, The German comet group - Analysis of past comet apparitions, http://kometen.fg-vds.de/oldause.htm

Królikowska, M., \& Dybczyński, P. A. 2010, MNRAS, 404, 1886

Marsden, B. G., \& Williams, G. V. 2008, Catalogue of Cometary Orbits 17th Edition (Cambridge, Mass.: Smithsonian Astrophysical Observatory)

Marsden, B. G., Sekanina, Z., \& Yeomans, D. K. 1973, AJ, 78, 211

Milam, S. N., Remijan, A. J., Womack, M., et al. 2006, ApJ, 649, 1169

Rickman, H. 2010, in Lecture Notes in Physics 790 (Berlin: Springer Verlag), eds. J. Souchay, \& R. Dvorak, 341

Sekanina, Z. 1988, AJ, 95, 911

Sekanina, Z. 2004, IAU Circ., 8330, 1

Sitarski, G. 1998, Acta Astron., 48, 547

Szutowicz, S., Królikowska, M., \& Sitarski, G. 2002a, Earth Moon and Planets, 90, 119

Szutowicz, S., Królikowska, M., \& Sitarski, G. 2002b, in Asteroids, Comets, and Meteors: ACM 2002, ed. B. Warmbein, ESA Spec. Publ., 500, 633

Tozzi, G. P., Boehnhardt, H., \& Lo Curto, G. 2003, A\&A, 398, L41

van Leeuwen, F. 2007, A\&A, 474, 653

Yabushita, S. 1996, MNRAS, 283, 347 